\title{
Diet and cognitive decline at middle age: the role of antioxidants
}

\author{
Astrid C. J. Nooyens ${ }^{1 *}$, Ivon E. J. Milder ${ }^{1}$, Boukje M. van Gelder ${ }^{1}$, H. Bas Bueno-de-Mesquita ${ }^{1,2,3,4}$, \\ Martin P. J. van Boxtel ${ }^{5}$ and W. M. Monique Verschuren ${ }^{1,6}$ \\ ${ }^{1}$ Centre for Nutrition, Prevention and Health Services, National Institute for Public Health and the Environment (RIVM), \\ PO Box 1, 3720 BA Bilthoven, The Netherlands \\ ${ }^{2}$ Department of Gastroenterology and Hepatology, University Medical Centre Utrecht (UMCU), Utrecht, The Netherlands \\ ${ }^{3}$ The School of Public Health, Imperial College London, London, UK \\ ${ }^{4}$ Department of Social and Preventive Medicine, Faculty of Medicine, University of Malaya, Kuala Lumpur, Malaysia \\ ${ }^{5}$ Department of Psychiatry and Neuropsychology, Maastricht University, Postbus 616, 6200 MD, Maastricht, The Netherlands \\ ${ }^{6}$ Center for Health Sciences and Primary Care, University Medical Center Utrecht (UMCU), Utrecht, The Netherlands
}

(Submitted 6 January 2014 - Final revision received 2 January 2015 - Accepted 11 February 2015 - First published online 8 April 2015)

\section{Abstract}

To assess the relationship between dietary intake of antioxidants (vitamin $\mathrm{C}$, vitamin $\mathrm{E}, \boldsymbol{\beta}$-carotene, lutein, flavonoids and lignans) and cognitive decline at middle age, analyses were performed on data from the population based Doetinchem Cohort Study. Habitual diet and cognitive function were assessed twice with a 5-year interval in 2613 persons aged 43-70 year at baseline (1995-2002). Diet was assessed with a validated 178-item semi-quantitative FFQ. Cognitive function was assessed with a neuropsychological test battery, consisting of the 15 Words Learning Test, the Stroop Test, the Word Fluency test, and the Letter Digit Substitution Test. Scores on global cognitive function, memory, processing speed, and cognitive flexibility were calculated. In regression analyses, quintiles of antioxidant intake were associated with change in cognitive domain scores. Results showed that higher lignan intake was linearly associated with less decline in global cognitive function $(P=0 \cdot 01)$, memory $(P<0 \cdot 01)$ and processing speed $(P=0.04)$, with about two times less declines in the highest $v$. the lowest quintile. In the lowest quintile of vitamin $\mathrm{E}$ intake, decline in memory was twice as fast as in all higher quintiles $(P<0 \cdot 01)$. Global cognitive decline in the highest lutein intake group was greater than in the lowest intake group $(P<0.05)$. Higher flavonoid intake was associated with greater decline in cognitive flexibility ( $P$ for trend $=0.04)$. Intakes of other antioxidants were not associated with cognitive decline. We conclude that within the range of a habitual dietary intake, higher intake of lignans is associated with less cognitive decline at middle age.

\section{Key words: Antioxidants: Cognitive decline: Cohort studies: Middle-aged populations}

Ageing-related neurodegenerative diseases can be due to failure of protective mechanisms caused by dietary deficiencies, for instance in antioxidants ${ }^{(1)}$. Antioxidants may help to neutralize tissue-damaging free radicals, which become more prevalent with age ${ }^{(2)}$. Therefore, higher dietary antioxidant intake may slow down the damaging effects of free radicals on neurons and thereby protect against neurodegenerative diseases, such as dementia.

This hypothesis has been tested in several studies associating dietary antioxidant intake with cognitive function: in a few studies ${ }^{(3-5)}$ with cognitive decline in the elderly ${ }^{(6-9)}$, and in a few others with incidence of dementia ${ }^{(10-13)}$. Results were varying, with no or favourable associations for higher antioxidant intake. Cross-sectional associations with cognitive function are not readily translated into cause and effect relationships. Associations with change in cognitive function in a relatively young and healthy population give better insight into potential preventive measures to slow down cognitive decline.Furthermore, it is possible that the elderly who developed clinical dementia during follow-up, already had had sub-clinical deteriorated cognitive function at baseline, which, in turn, might have influenced already the dietary intake of these elderly subjects. In addition, prevention of disease is more likely in a younger cohort ${ }^{(11)}$. Therefore, it is important to study associations between antioxidant intake and cognitive decline in a relative young and healthy population.

In three of the above-mentioned studies, the Dutch Rotterdam study $(55+\text { years })^{(12)}$, the French Supplementation in Vitamins and Mineral Antioxidants (SU.VI.MAX) study $(45-60 \text { years })^{(3,4)}$, and the US Atherosclerosis Risk in Communities (ARIC) study (48-67 years) ${ }^{(5)}$, dietary antioxidant intake

Abbreviations: ARIC, Atherosclerosis Risk in Communities; SU.VI.MAX, Supplementation in Vitamins and Mineral Antioxidants.

*Corresponding author: Dr A. C. J. Nooyens, fax +31 30274 4407, email astrid.nooyens@rivm.nl 
was examined in relation to cognitive function of middle-aged populations. However, in these studies dietary intake was assessed only once, and in two of the studies ${ }^{(3-5)}$, cognitive function too was measured only once. The present study, in contrast, assessed dietary intake and cognitive function both at baseline and at follow-up. Therefore, it was possible to achieve a more robust measure of habitual (long-term) dietary intake and to study the related cognitive decline as well. It was hypothesized for the study that persons with higher habitual intake of dietary antioxidants would experience less cognitive decline at middle age.

\section{Subjects and methods}

\section{Population}

The Doetinchem Cohort Study ${ }^{(14)}$ is an ongoing prospective study initially carried out among a representative sample of 12405 men and women aged 20-59 years during the first examination (1987-91). A random sample of 7769 of these participants were re-invited for the second (1993-7), the third (1998-2002) and the fourth (2003-7) examinations. Response rates varied from 75 to $79 \%$. All participants gave written informed consent. The study was approved by the external Medical Ethics Committee of the Dutch TNO Research Institute according to the guidelines of the Helsinki Declaration. Details on the Doetinchem Cohort Study can be found elsewhere ${ }^{(14)}$.

In 1995, the Doetinchem Cohort Study introduced cognitive testing for participants aged 45 years and older. A total of 3350 respondents aged $43-70$ years participated for the first time in cognitive testing between 1995 and 2002 ( = baseline measurement cognitive function), and 2690 of them (80\%) participated again in cognitive testing 5 years later. Participants who had reported to have had a stroke $(n 77)$ were excluded, leaving a total of 2613 participants (1288 men and 1325 women) who provided two cognitive measurements for the present analyses.

\section{Measurements}

Cognitive tests. The neuropsychological test battery consisted of four subtests: the 15 Words Learning Test, the Stroop Test, the Word Fluency test, and the Letter Digit Substitution Test. These tests are described in more detail elsewhere ${ }^{(15)}$. The tests are sensitive to age, including the middle-age range. They are robust in detecting age-related impairment, even at middle age, and have been used in several other large-scale studies on cognitive function ${ }^{(16-18)}$

Antioxidant intake. At baseline and at follow-up, a validated self-administered semi-quantitative FFQ, developed for the European Investigation into Cancer and Nutrition (EPIC), was used to assess the habitual consumption of 178 food items during the previous year ${ }^{(19,20)}$. Dietary intakes of vitamin $C$, vitamin $E$ and $\beta$-carotene were calculated based on the contemporary Dutch Food Composition Database ${ }^{(21)}$. Dietary intakes of flavonoids (flavonols, flavons, and catechins) and the most important lignans (lariciresinol, pinoresinol, secoisolariciresinol, and matairesinol) were based on food analyses carried out specifically for these substances $^{(22-26)}$. Dietary intake of lutein was calculated based on the online version of the Dutch Food Composition Database (2013) ${ }^{(27)}$. Supplemental intakes of vitamins C and E were assessed based on the questionnaire data (self-report).

Covariates. Information on demographic characteristics (e.g. age, educational level), lifestyle factors (e.g. smoking, physical activity), medical history of chronic diseases and medication used was collected using standardized questionnaires at baseline and at follow-up. Educational level was assessed as the highest level reached over follow-up and classified into five categories, ranging from primary school to higher vocational or university education. Smoking status was defined as being a never-smoker, ex-smoker (quit before baseline), quitter (quit between baseline and followup), starter (between baseline and follow-up) or persistent smoker over follow-up, and the number of life-long pack years smoked was computed. Based on the physical activity questionnaire $^{(28)}$, physical activity was classified into four categories: inactive, moderately inactive, moderately active, and active (Cambridge Physical Activity Index) ${ }^{(29)}$. Based on the $\mathrm{FFQ}^{(19,20)}$ total energy intake was computed on the basis of nutritional values in the Dutch Food Composition Database $^{(21)}$. Mental quality of life was assessed with the Dutch version ${ }^{(30)}$ of the SF-36 $6^{(31)}$. During the physical examination at the research centre, height, weight, waist circumference and blood pressure were measured, and nonfasting blood samples were obtained, to determine serum total and HDL cholesterol ${ }^{(14)}$.

\section{Statistical methods}

Mean intakes of the specific antioxidants were computed over baseline and follow-up, in order to obtain more stable estimates of habitual intakes. Mean intakes were subsequently classified into quintiles. For vitamin $\mathrm{C}$ and vitamin $\mathrm{E}$, supplement users were classified in the highest quintile of intake.

For each cognitive test, a standardized $z$-score was computed at baseline and at follow-up, on the basis of the means and standard deviations of the test scores at baseline. Based on the neuropsychological test battery were computed a global cognitive function score (all tests) and scores on three specific cognitive domains, viz., memory function (the 15 Words Learning Test), speed of cognitive processes (the Stroop Test and the Letter Digit Substitution Test), and cognitive flexibility (i.e. higher order information processing; the Stroop Test) ${ }^{(15)}$.

Domain-specific cognitive functions at baseline could be assessed for 2581 (global cognitive function) up to 2604 (cognitive flexibility) participants. Change in cognitive function score (follow-up score minus baseline score) could be calculated for 2559 (global cognitive function) up to 2597 (memory function) participants.

In addition, mean cognitive decline over follow-up was computed for each quintile of antioxidant intake, and compared between quintiles using ANCOVA (PROC GLM in SAS; SAS Institute, Inc.). For $P$ for trend analyses, the participants were assigned the median for their quintile, and then linear regression analyses were performed on these medians in relation to change in cognitive function ${ }^{(32)}$. In all the analyses done, 
adjustments were made for age, sex, level of education, all other antioxidant intakes (including vitamin $\mathrm{C}$ and vitamin $\mathrm{E}$ supplement use), total energy intake, smoking (status and pack years), physical activity, waist circumference, mental quality of life, and baseline cognitive function. The selection of potential covariants was based on past literature and theoretical considerations. These covariants were then tested in the regression models. Potential confounders that did not influence the association between antioxidant intake and cognitive decline were not included in the presented models. Diabetes, heart disease, hypertension, hypercholesterolemia and hormone replacement therapy (in women) did not essentially change associations and were therefore not included in the analyses.

We tested for interaction between antioxidant intake and subgroups of sex (men/women), age (younger $v$. older than 60 years), level of education (low/high), or smoking (smoker at baseline yes/no). No interaction effects were observed at $P<0 \cdot 10$. Also, we tested whether associations were different when we took along total vitamin $\mathrm{C}$ and vitamin $\mathrm{E}$ intake (total of dietary and supplemental intake), instead of dietary intake adjusted for supplemental intake. Since associations were not essentially different, results of only dietary intake are considered in the present paper. Associations or differences between quintiles were regarded as significant at $P<0 \cdot 05$. All analyses were performed using SAS 9.3 (SAS Institute, Inc.).

\section{Results}

Participants were on average 55 years old at baseline. Mean follow-up was 5.0 (SD 0.1) years. Persons who scored below the mean on global cognitive function were older, more often male and less educated than persons who scored above the mean (Table 1). Mean intakes of vitamin C, $\beta$-carotene, flavonoids and lignans were marginally lower, and mean intake of vitamin E was slightly higher in the group that scored below the mean on global cognitive function compared to the group that scored above the mean on global cognitive function.

Median dietary intakes in the lowest and the highest quintile of intake (excluding supplemental intake) were 65 and

Table 1. Baseline characteristics of the study population, by low or high global cognitive function at baseline (Doetinchem Cohort Study 1995-2007)

(Mean values and standard deviations; percentages)

\begin{tabular}{|c|c|c|c|c|c|c|}
\hline & \multicolumn{2}{|c|}{ All $(n$ 2613) } & \multicolumn{2}{|c|}{$\begin{array}{l}\text { Baseline global } \\
\text { cognitive function } \\
\text { below the mean } \\
(n 1255)^{\star}\end{array}$} & \multicolumn{2}{|c|}{$\begin{array}{l}\text { Baseline global } \\
\text { cognitive function } \\
\text { above the mean } \\
(n 1326)^{\star}\end{array}$} \\
\hline & Mean & SD & Mean & SD & Mean & SD \\
\hline Age (years) & $55 \cdot 3$ & $6 \cdot 9$ & $57 \cdot 2$ & $7 \cdot 1$ & $53 \cdot 4$ & $6 \cdot 1$ \\
\hline Sex (\% women) & $50 \cdot 7$ & & 44.5 & & $56 \cdot 9$ & \\
\hline Level of education (\% low educated) $\dagger$ & $32 \cdot 0$ & & $48 \cdot 7$ & & $16 \cdot 1$ & \\
\hline \multicolumn{7}{|l|}{ Cognitive function domain scores ( $z$-scores) $\ddagger$} \\
\hline Global cognitive function ( $n$ 2581) & 0.00 & $0 \cdot 72$ & -0.59 & 0.45 & 0.57 & 0.42 \\
\hline Memory function ( $n$ 2602) & 0.00 & 0.94 & -0.62 & 0.73 & 0.60 & 0.70 \\
\hline Speed of cognitive processes ( $n$ 2598) & 0.00 & 0.84 & -0.49 & 0.73 & 0.47 & 0.64 \\
\hline Cognitive flexibility ( $n$ 2604) & 0.00 & 1.00 & -0.54 & 0.93 & 0.51 & 0.77 \\
\hline Total energy intake (MJ/d) & $9 \cdot 0$ & $2 \cdot 3$ & $9 \cdot 1$ & $2 \cdot 4$ & $8 \cdot 8$ & $2 \cdot 2$ \\
\hline Vitamin C intake $(\mathrm{mg} / \mathrm{d}) \S$ & 111 & 45 & 108 & 46 & 113 & 44 \\
\hline Vitamin E intake $(\mathrm{mg} / \mathrm{d}) \S$ & 13 & 5 & 13 & 5 & 13 & 5 \\
\hline$\beta$-Carotene intake $(\mu \mathrm{g} / \mathrm{d})$ & 1480 & 593 & 1475 & 649 & 1489 & 537 \\
\hline Lutein $(\mu \mathrm{g} / \mathrm{d})$ & 1796 & 784 & 1844 & 830 & 1752 & 731 \\
\hline Flavonoid intake (mg/d) & 62 & 43 & 59 & 42 & 64 & 43 \\
\hline Lignan intake $(\mu \mathrm{g} / \mathrm{d})$ & 1017 & 262 & 997 & 265 & 1038 & 256 \\
\hline Vitamin C supplements (\% users) & $19 \cdot 0$ & & $17 \cdot 1$ & & $21 \cdot 1$ & \\
\hline Vitamin E supplements (\% users) & 4.3 & & $4 \cdot 1$ & & 4.6 & \\
\hline Smoking (\%) & $22 \cdot 0$ & & 21.9 & & $22 \cdot 0$ & \\
\hline Physical activity (\% inactive) $\|$ & $25 \cdot 0$ & & $26 \cdot 5$ & & 23.4 & \\
\hline Alcohol consumption (glasses/d) & 1.4 & 1.6 & $1 \cdot 3$ & 1.6 & 1.4 & 1.5 \\
\hline Systolic blood pressure (mmHg) & 131 & 18 & 134 & 18 & 128 & 17 \\
\hline Hypertension (\%) & 35.5 & & $40 \cdot 8$ & & $30 \cdot 1$ & \\
\hline Total cholesterol $(\mathrm{mmol} / \mathrm{l})$ & $5 \cdot 85$ & 1.01 & $5 \cdot 88$ & 1.03 & $5 \cdot 81$ & 0.99 \\
\hline \multicolumn{7}{|l|}{ HDL cholesterol (mmol/l) } \\
\hline Men & 1.22 & 0.32 & $1 \cdot 21$ & 0.33 & 1.23 & 0.32 \\
\hline Women & 1.53 & 0.39 & 1.48 & 0.37 & 1.57 & 0.39 \\
\hline \multicolumn{7}{|l|}{ Waist circumference $(\mathrm{cm})$} \\
\hline Men & $98 \cdot 7$ & $9 \cdot 2$ & $99 \cdot 9$ & $9 \cdot 5$ & $97 \cdot 2$ & $8 \cdot 6$ \\
\hline Women & 89.5 & 11.0 & $92 \cdot 1$ & 11.3 & 87.5 & $10 \cdot 3$ \\
\hline
\end{tabular}

${ }^{*}$ A valid score on global cognitive function was available for 2581 of the 2613 participants.

†Primary school or lower vocational education as highest attained level.

$\ddagger$ Higher score $=$ better.

§ Excluding supplement intake.

ๆ Being classified as inactive or moderately inactive, according to the Cambridge Physical Activity Index ${ }^{(28)}$ 
$148 \mathrm{mg} / \mathrm{d}$ for vitamin C, 8 and $18 \mathrm{mg} / \mathrm{d}$ for vitamin E, 952 and $2070 \mu \mathrm{g} / \mathrm{d}$ for $\beta$-carotene, 19 and $114 \mathrm{mg} / \mathrm{d}$ for flavonoids, 1039 and $2617 \mu \mathrm{g} / \mathrm{d}$ for lutein, and 733 and $1350 \mu \mathrm{g} / \mathrm{d}$ for lignans.

Higher intake of lignans was associated with less decline in global cognitive function ( $P$ for trend $=0 \cdot 01$ ), memory $(P$ for trend $<0.01)$ and speed of cognitive processes $(P$ for trend $=0.05$ ) over follow up. Persons in the lowest quintile of lignan intake had a 3.5 times greater decline in global cognitive function, a six times greater decline in memory, and a two times greater decline in speed of cognitive processes compared to persons in the highest quintile (Table 2).
However, these results were partly driven by baseline differences in cognitive function between the quintiles of lignan intake. To illustrate this, baseline and follow-up levels of cognitive function in each quintile of lignan intake are presented in Fig. 1. This figure shows linear associations between lignan intake and all cognitive domains at baseline, as well as decline in all cognitive domains over follow-up. Based on these data, cognitive decline in the lowest quintile of lignan intake is about twice the decline in the highest quintile. For the other antioxidants, the observed associations were not consistent. No associations between intakes of vitamin $C$ and $\beta$-carotene and cognitive decline were observed. For vitamin E, no linear

Table 2. Adjusted† associations between habitual intake of antioxidants and change in cognitive function ( $z$-scores) (Doetinchem Cohort Study, 1995-2007)

(Median values and ranges; mean changes)

\begin{tabular}{|c|c|c|c|c|c|c|c|}
\hline & & \multicolumn{5}{|c|}{ Quintiles of intake } & \multirow[b]{2}{*}{$P$ for trend $\ddagger$} \\
\hline & & I (low) & II & III & IV & V (high) & \\
\hline \multirow[t]{7}{*}{ Vitamin C } & Median consumption in category $(\mathrm{mg} / \mathrm{d}) \S$ & 65 & 87 & 108 & 128 & 148 & \\
\hline & Range & $20-76$ & $76-97$ & $98-118$ & $118-144$ & $41-308$ & \\
\hline & $n$ & 416 & 430 & 433 & 415 & 919ף & \\
\hline & Global & -0.09 & -0.04 & -0.12 & -0.07 & $-0 \cdot 10$ & 0.29 \\
\hline & Memory & -0.13 & $-0 \cdot 10$ & -0.19 & -0.08 & -0.13 & 0.85 \\
\hline & Speed & -0.11 & -0.10 & -0.16 & -0.14 & -0.12 & 0.51 \\
\hline & Flexibility & -0.06 & 0.00 & -0.13 & -0.10 & $-0 \cdot 10$ & 0.07 \\
\hline \multirow[t]{7}{*}{ Vitamin $\mathrm{E}$} & Median consumption in category (mg/d) & 8 & 11 & 12 & 14 & 18 & \\
\hline & Range & $5-10$ & $10-11$ & $11-13$ & $13-16$ & $6-39$ & \\
\hline & $n$ & 498 & 512 & 497 & 497 & 6099 & \\
\hline & Global & $-0 \cdot 12$ & -0.08 & -0.09 & -0.07 & -0.08 & 0.26 \\
\hline & Memory & -0.22 & $-0 \cdot 10^{\star \star}$ & $-0.11^{*}$ & $-0.11^{*}$ & $-0.09^{*}$ & 0.07 \\
\hline & Speed & -0.13 & -0.10 & -0.15 & -0.12 & -0.13 & 0.64 \\
\hline & Flexibility & $-0 \cdot 10$ & -0.09 & -0.10 & -0.07 & -0.06 & 0.28 \\
\hline \multirow[t]{7}{*}{$\beta$-Carotene } & Median consumption in category $(\mu \mathrm{g} / \mathrm{d})$ & 952 & 1205 & 1419 & 1653 & 2070 & \\
\hline & Range & $290-1093$ & $1093-1321$ & $1321-1526$ & $1526-1801$ & $1803-5848$ & \\
\hline & $n$ & 522 & 523 & 523 & 523 & 522 & \\
\hline & Global & -0.08 & -0.11 & -0.09 & -0.07 & -0.08 & 0.49 \\
\hline & Memory & -0.10 & -0.16 & -0.16 & -0.07 & -0.14 & 0.98 \\
\hline & Speed & -0.09 & -0.13 & -0.14 & -0.14 & -0.12 & 0.46 \\
\hline & Flexibility & -0.06 & -0.11 & -0.05 & -0.10 & -0.10 & 0.45 \\
\hline \multirow[t]{7}{*}{ Lutein } & Median consumption in category $(\mu \mathrm{g} / \mathrm{d})$ & 1039 & 1394 & 1678 & 2009 & 2617 & \\
\hline & Range & $372-1249$ & $1249-1538$ & $1538-1835$ & $1835-2246$ & $2247-5713$ & \\
\hline & $n$ & 522 & 523 & 523 & 523 & 522 & \\
\hline & Global & -0.06 & -0.09 & $-0 \cdot 10$ & -0.08 & $-0.11^{*}$ & $0 \cdot 10$ \\
\hline & Memory & -0.10 & -0.13 & -0.15 & -0.11 & -0.13 & 0.60 \\
\hline & Speed & -0.12 & -0.12 & -0.17 & -0.11 & -0.11 & 0.59 \\
\hline & Flexibility & -0.07 & -0.07 & -0.08 & -0.09 & -0.12 & 0.21 \\
\hline \multirow[t]{7}{*}{ Flavonoids } & Median consumption in category (mg/d) & 19 & 36 & 53 & 75 & 114 & \\
\hline & Range & $3-27$ & $27-43$ & $43-63$ & $63-91$ & $91-272$ & \\
\hline & $n$ & 522 & 523 & 523 & 523 & 522 & \\
\hline & Global & -0.08 & -0.08 & -0.05 & $-0 \cdot 10$ & -0.11 & $0 \cdot 18$ \\
\hline & Memory & -0.11 & -0.11 & -0.09 & -0.16 & -0.15 & 0.35 \\
\hline & Speed & -0.12 & -0.11 & -0.11 & -0.14 & -0.15 & 0.27 \\
\hline & Flexibility & -0.04 & -0.08 & -0.05 & -0.11 & $-0.14^{\star}$ & 0.04 \\
\hline \multirow{7}{*}{ Lignans } & Median consumption in category $(\mu \mathrm{g} / \mathrm{d})$ & 733 & 894 & 1013 & 1143 & 1350 & \\
\hline & Range & $415-822$ & $822-951$ & $951-1078$ & $1079-1231$ & $1231-2337$ & \\
\hline & $n$ & 522 & 523 & 523 & 523 & 522 & \\
\hline & Global & -0.14 & -0.11 & $-0.07^{*}$ & -0.08 & $-0.04^{\star}$ & 0.01 \\
\hline & Memory & -0.24 & -0.16 & $-0 \cdot 10^{\star \star}$ & $-0.08^{\star \star}$ & $-0.04^{\star \star}$ & $<0.01$ \\
\hline & Speed & -0.15 & -0.16 & -0.11 & -0.13 & -0.07 & 0.05 \\
\hline & Flexibility & -0.14 & -0.10 & -0.07 & -0.07 & -0.03 & 0.06 \\
\hline
\end{tabular}

Value was significantly different from that of lowest quintile: ${ }^{*} P<0.05,{ }^{\star *} P<0.01$

† Mean changes in quintiles and $P$ for trend are adjusted for age, sex, level of education, baseline cognitive function, all other antioxidant intakes (including supplement use), total energy intake, smoking, physical activity, waist circumference, and mental quality of life.

$\ddagger P$ for trend is calculated over medians of dietary antioxidant intake in the quintiles.

$\S$ Excluding supplement intake.

If For vitamin $\mathrm{C}$ and vitamin $\mathrm{E}$, supplement users are classified in the highest quintile of intake. 


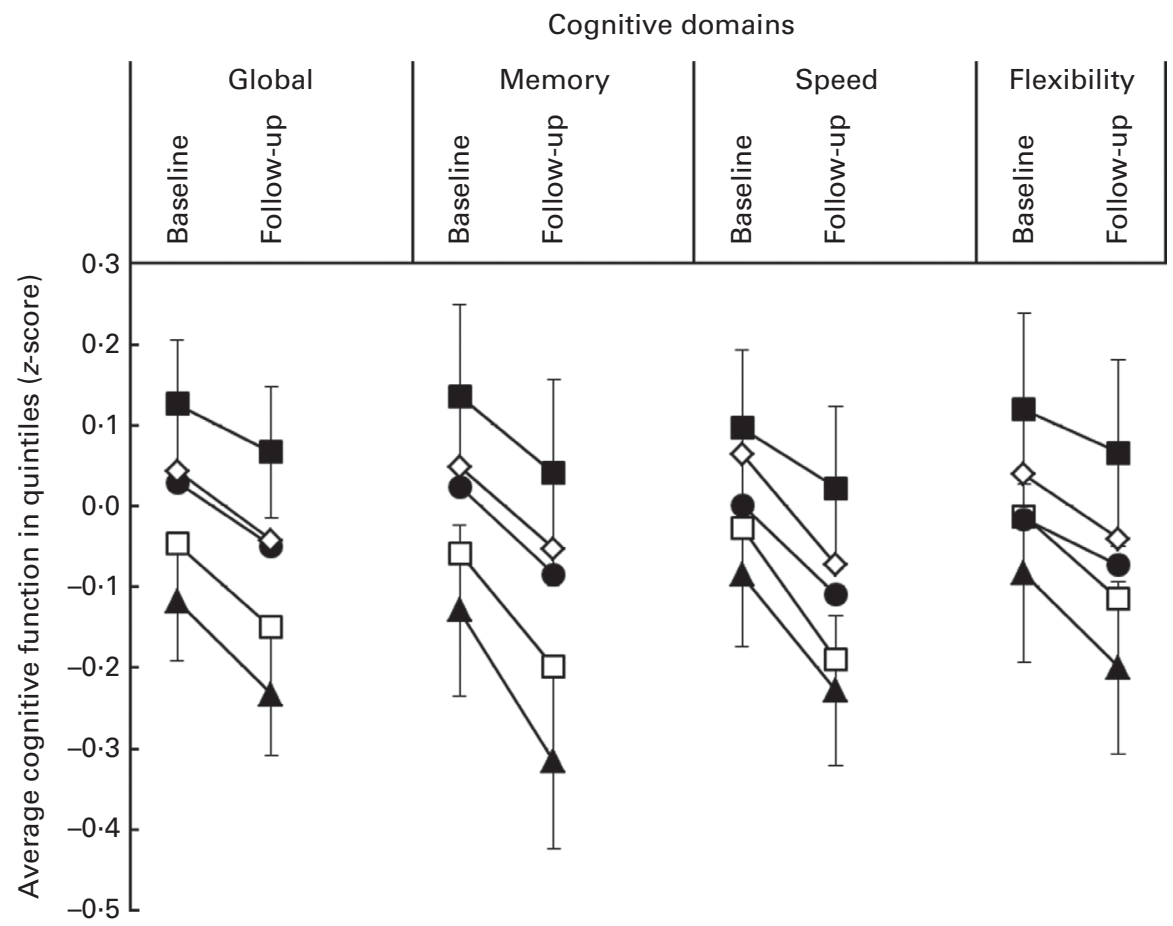

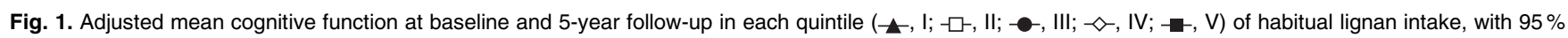
confidence limits for lowest (I) and highest (V) quintiles (Doetinchem Cohort Study 1995-2007). Mean cognitive function is adjusted for age, sex, level of education, all other antioxidant intakes (including supplement use), total energy intake, smoking, physical activity, waist circumference, and mental quality of life.

trend was observed, but memory of persons in the lowest quintile declined about two times greater than that of persons in all other quintiles $(P<0 \cdot 01)$. Participants with a higher intake of flavonoids showed greater decline in cognitive flexibility ( $P$ for trend $=0 \cdot 04$ ). For lutein, global cognitive decline for the highest intake group was greater than the decline in the lowest intake group $(P<0.05)$ (Table 2$)$.

\section{Discussion}

In the present study, habitual dietary intake of antioxidants (vitamin $\mathrm{C}$, vitamin $\mathrm{E}, \boldsymbol{\beta}$-carotene, lutein, flavonoids and lignans) was examined in relation to change in cognitive function. Higher lignan intake was strikingly associated with lower decline in global cognitive function, memory function, and speed of cognitive processes. For vitamin $\mathrm{E}$, greater memory decline was observed in the lowest quintile compared to all other quintiles. None of the other antioxidants was consistently associated with cognitive decline.

The major strengths of the present study are the prospective design, the relatively young and healthy participants and the use of a sensitive cognitive test battery. It investigated habitual dietary intake, not short term supplementation with certain micronutrients as performed in most trials. In addition to the extensive FFQ, the Doetinchem Cohort Study has an extensive spectrum of variables assessed during the repeated measurements, making it possible to adjust for a wide range of potential confounders. In a recent review, Crichton et al. ${ }^{(33)}$ recommended that future studies should utilise thorough neuropsychological testing across a range of cognitive functions, include younger individuals, have multiple follow ups, and assess multiple classes of antioxidants'. The present study had satisfied all these recommendations.

One of the drawbacks of long-term cohort studies such as the present one is the dropping-out of participants enrolled at the beginning of the study. The $80 \%$ response at followup of the present study is quite high; nevertheless it leads to some selection of a healthier sample. The results of such a bias may be that the associations observed underestimate the 'true' associations.

In three studies viz., the Dutch Rotterdam study $(55+\text { years })^{(12)}$, the French SU.VI.MAX study (45-60 years $)^{(3,4)}$, and the US ARIC study (48-67 years) ${ }^{(5)}$, dietary antioxidant intake was investigated in relation to cognitive function in a middle-aged population. In the Rotterdam study, higher dietary intake of vitamin C and E at middle age was associated with lower incidence of Alzheimer's disease over the next 6 years. Among smokers, higher flavonoid and $\beta$-carotene intake were associated with lower incidence of Alzheimer's disease ${ }^{(12)}$. We did not observe in the present study such associations between dietary intake of these antioxidants and cognitive decline at middle age, although low vitamin $\mathrm{E}$ intake was associated with greater memory decline. In the SU.VI.MAX study, cognitive function was measured at follow-up, 13 years after the assessment of dietary intake ${ }^{(3,4)}$. Supplementation with antioxidant vitamins and minerals was positively associated with verbal memory (in non-smokers and persons with a low serum vitamin C status only) and executive function ${ }^{(4)}$. However, supplemental antioxidant intakes were much higher than what can be obtained from 
a habitual diet. In the same study, higher total flavonoid intake at baseline was associated with better verbal memory function, but not (or mostly oppositely) with executive function, while lignan intake was associated with better cognitive function in the basic model only (adjusted for age and sex $)^{(3)}$. We also observed in the present study a detrimental association between higher flavonoid intake and decline in cognitive flexibility, but more strikingly, we observed strong beneficial associations between higher lignan intake and cognitive decline (especially memory) over follow-up. Differences in results between the present study and the SU.VI.MAX study may be due to different nutritional databases. In the SU.VI.MAX study more subgroups of flavonoids were taken along and intakes of catechins, flavones and flavonols were much higher compared with the present study. In contrast, total intake of lignans in the SU.VI.MAX was about half of the intake of lignans in the present study. Finally, in the ARIC study, cross-sectional associations of carotenoids, vitamin $\mathrm{C}$ and vitamin $\mathrm{E}$ intake and cognitive function were evaluated, but no consistent associations were observed ${ }^{(5)}$.

Two reviews on the association between dietary antioxidants and cognitive decline in older populations concluded that there is a possible, but no consistent protective association between dietary antioxidants and cognitive decline ${ }^{(33,34)}$. In a meta-analysis, pooled relative risks suggested that higher intakes of vitamin $\mathrm{C}$, vitamin $\mathrm{E}$ and $\beta$-carotene could lower the risk of Alzheimer's disease ${ }^{(35)}$. Most pronounced beneficial associations were observed for vitamin $\mathrm{E}$, followed by vitamin $\mathrm{C}$ and $\beta$-carotene; this is in line with the results of the present study.

Smoking increases strongly oxidative stress, and it has been hypothesized that relatively weak associations with antioxidants may not be found in the case of smokers ${ }^{(36)}$. However, in the Rotterdam study, associations were most pronounced in smokers ${ }^{(12)}$. In the present study, results were not different for smokers and non-smokers.

The present study did not find (strong) associations between habitual dietary intakes of specific antioxidants and cognitive decline (except for lignans). This may be because there is no association between antioxidant intake and cognitive decline at middle age, as was also observed in other studies in both middle-aged and elderly populations ${ }^{(37-40)}$. However, some methodological considerations should be pointed out. It may be that the variation in the amount of antioxidant intake was too small to observe an effect. In a recent review on vitamin $\mathrm{C}$ for the prevention of age-related cognitive decline and Alzheimer's disease, it was concluded that avoiding vitamin $\mathrm{C}$ deficiency is likely to be more beneficial than taking supplemental intake on top of a normal, healthy diet ${ }^{(41)}$. In the present study, only few persons had a suboptimal vitamin $\mathrm{C}$ intake, but low vitamin $\mathrm{E}$ intake was associated with stronger cognitive decline. Higher dietary vitamin $\mathrm{E}$ intake did not have added value, but users of vitamin E supplements ( $4.3 \%$ of the participants) had lower decline in memory function $(P=0.01)$ and global cognitive function $(P=0.04)$ than non-users (data not shown). Possibly the duration of the follow-up might have been too short to detect an association. The observed association between higher flavonoid intake and greater decline in cognitive flexibility was not expected, and observed for only one of the cognitive domains. Although this observation was consistent with results of the SU.VI.MAX study ${ }^{(3)}$, this might be a chance finding.

Lutein which is mainly found in green vegetables is the predominant carotenoid in human brain tissue and may play a role in the maintenance of cognitive abilities through its antioxidation and anti-inflammation properties ${ }^{(42)}$. However, the present study did not find beneficial associations between lutein intake and cognitive decline. Moreover, the global cognitive decline for the highest intake group was greater than the decline in the lowest intake group. Also at baseline, cognitive performance in the highest lutein intake group was statistically significantly lower than that in the lower intake groups for speed and flexibility (data not shown). These results are not in line with conclusions of a recent review on the role of lutein in cognitive function. Possibly, lutein supplementation is needed to find such beneficial associations $^{(43)}$

The present authors observed consistent beneficial associations between habitual lignan intake and cognitive decline, especially memory decline. Differences between the lowest and the highest quintile of lignan intake were similar to or even larger than the difference in cognitive decline between smokers and non-smokers ${ }^{(44)}$. Moreover, already at baseline a statistically significant positive trend in memory function and global cognitive function was observed over the quintiles of lignan intake (see also Fig. 1). Lignan intake had strongest association with memory decline as opposed to associations with decline in other cognitive domains. A statistical reason for this effect could be the observation that cognitive decline and its standard deviation, expressed in $z$-scores, were largest for memory decline. However, higher lignan intake was already found associated with better memory function at baseline (when all functions were standardized into $z$-scores). Therefore, results suggest a possible protective effect of higher lignan intake on cognitive decline, which is most pronounced for age-related memory decline. Most important sources of lignans were beverages (tea, coffee), vegetables (cabbages), and nuts and seeds ${ }^{(45)}$. Based merely on observational data, we cannot attribute the beneficial effect to lignans: lignan intake may just have been a proxy measure for a cognitively healthy diet of the present population. However, there are also indications that a higher lignan intake may be responsible for any cognition-preserving effects. The studied plant lignans are the ones that are most efficiently converted into enterolignans by the intestinal microflora ${ }^{(46)}$. Enterolignans possess several biological activities, e.g. antioxidant and oestrogen-like activities by which they may reduce the risk of chronic diseases ${ }^{(47)}$.

Therefore, beneficial effects of antioxidants as found in animal studies ${ }^{(48)}$ could actually have been the result of higher lignan intake: the high antioxidant fruits (blueberry, boysenberry, cranberry, black currant, strawberry, dried plums, grape) and vegetable (spinach) as used in these studies are also rich sources of lignans and other phyto-oestrogens ${ }^{(26,49)}$. Also a cross-sectional study on postmenopausal women 
observed a beneficial association between lignan intake and cognitive function ${ }^{(50)}$. Compared with, e.g. vitamins $\mathrm{C}$ and $\mathrm{E}$, lignans contribute only a little to total antioxidant intake. Therefore, we hypothesize that the phyto-oestrogen capacity of lignans may be responsible for the observed beneficial effects. In earlier studies, consistent beneficial associations between the consumption of cabbage ${ }^{(15)}$, nuts ${ }^{(15)}$, and red wine $^{(51)}$ and cognitive decline were observed. These foods are typically rich sources of lignans and stilbenes (synthesized from resveratrol), another family of phyto-oestrogens ${ }^{(49)}$.

In conclusion, within the intake range of a habitual diet, most antioxidants were not consistently associated with cognitive decline over time. In contrast, higher habitual intake of lignans was consistently associated with lower cognitive decline at middle age. If confirmed by further studies, elevation of the amount of habitual consumption of foods rich in lignans could be one of the future nutritional recommendations for reducing cognitive decline at middle age; and it may eventually delay or prevent the onset of dementia.

\section{Acknowledgements}

The authors thank the respondents, the epidemiologists and fieldworkers of the Municipal Health Service in Doetinchem for their contribution to the data collection for this study. Principal investigator was Dr W. M. M. V.; logistic management was provided by J. Steenbrink and P. Vissink, and administrative support by E. P. van der Wolf. Data management was done by A. Blokstra, A. W. D. van Kessel and P. E. Steinberger. We acknowledge the works of Dr I. C. Arts and Dr M. G. L. Hertog in the determination of the content of several flavonoids in foods.

The present study was financially supported with a grant from the Internationale Stichting Alzheimer Onderzoek (ISAO, grant no. 08551). The Doetinchem Cohort Study is financially supported by the Ministry of Public Health, Welfare and Sport of the Netherlands and the National Institute for Public Health and Environment. The data up to and including 1997 and the dietary assessment methodology were additionally financially supported by the Europe Against Cancer Programme of the European Commission (DG SANCO). Funding agencies had no role in the design, analysis or writing of this article.

Contributions of authors were as follows: All authors were involved in interpreting the findings and reviewed drafts of the manuscript. A. C. J. N. performed the analyses of the data and took the lead in the writing of the article; W. M. M. V. supervised the data collection, analyses, and preparation of the article; H. B. B.-d.-M. supervised the development of the dietary assessment method and co-supervised the dietary data collection.

None of the authors has any conflict of interest.

\section{References}

1. Bourre JM (2006) Effects of nutrients (in food) on the structure and function of the nervous system: update on dietary requirements for brain. Part 1: micronutrients. $J$ Nutr Health Aging 10, 377-385.

2. McDaniel MA, Maier SF \& Einstein GO (2003) "Brainspecific" nutrients: a memory cure? Nutrition 19, 957-975.

3. Kesse-Guyot E, Fezeu L, Andreeva VA, et al. (2012) Total and specific polyphenol intakes in midlife are associated with cognitive function measured 13 years later. J Nutr 142, 76-83.

4. Kesse-Guyot E, Fezeu L, Jeandel C, et al. (2011) French adults' cognitive performance after daily supplementation with antioxidant vitamins and minerals at nutritional doses: a post hoc analysis of the Supplementation in Vitamins and Mineral Antioxidants (SU.VI.MAX) trial. Am J Clin Nutr 94, 892-899.

5. Peacock JM, Folsom AR, Knopman DS, et al. (2000) Dietary antioxidant intake and cognitive performance in middleaged adults. The Atherosclerosis Risk in Communities (ARIC) study investigators. Public Health Nutr 3, 337-343.

6. Letenneur L, Proust-Lima C, Le Gouge A, et al. (2007) Flavonoid intake and cognitive decline over a 10 -year period. Am J Epidemiol 165, 1364-1371.

7. Devore EE, Kang JH, Stampfer MJ, et al. (2010) Total antioxidant capacity of diet in relation to cognitive function and decline. Am J Clin Nutr 92, 1157-1164.

8. Kalmijn S, Feskens EJ, Launer LJ, et al. (1997) Polyunsaturated fatty acids, antioxidants, and cognitive function in very old men. Am J Epidemiol 145, 33-41.

9. Morris MC, Evans DA, Bienias JL, et al. (2002) Vitamin E and cognitive decline in older persons. Arch Neurol 59, $1125-1132$.

10. Commenges D, Scotet V, Renaud S, et al. (2000) Intake of flavonoids and risk of dementia. Eur J Epidemiol 16, 357-363.

11. Luchsinger JA, Tang MX, Shea S, et al. (2003) Antioxidant vitamin intake and risk of Alzheimer disease. Arch Neurol 60, 203-208.

12. Engelhart MJ, Geerlings MI, Ruitenberg A, et al. (2002) Dietary intake of antioxidants and risk of Alzheimer disease. JAMA 287, 3223-3229.

13. Morris MC, Evans DA, Bienias JL, et al. (2002) Dietary intake of antioxidant nutrients and the risk of incident Alzheimer disease in a biracial community study. JAMA 287, $3230-3237$.

14. Verschuren WMM, Blokstra A, Picavet HSJ, et al. (2008) Cohort profile: the Doetinchem Cohort Study. Int J Epidemiol 37, 1236-1241.

15. Nooyens AC, Bueno-de-Mesquita HB, van Boxtel MP, et al. (2011) Fruit and vegetable intake and cognitive decline in middle-aged men and women: the Doetinchem Cohort Study. Br J Nutr 106, 752-761.

16. de Groot JC, de Leeuw FE, Oudkerk M, et al. (2000) Cerebral white matter lesions and cognitive function: the Rotterdam Scan study. Ann Neurol 47, 145-151.

17. Møller JT, Cluitmans P, Rasmussen LS, et al. (1998) Long-term postoperative cognitive dysfunction in the elderly ISPOCD1 study. ISPOCD investigators. International study of PostOperative Cognitive Dysfunction. Lancet 351, 857-861.

18. Durga J, van Boxtel MP, Schouten EG, et al. (2007) Effect of 3 -year folic acid supplementation on cognitive function in older adults in the FACIT trial: a randomised, double blind, controlled trial. Lancet 369, 208-216.

19. Ocké MC, Bueno-de-Mesquita HB, Goddijn HE, et al. (1997) The Dutch EPIC food frequency questionnaire. I. Description of the questionnaire, and relative validity and reproducibility for food groups. Int J Epidemiol 26, Suppl. 1, S37-S48.

20. Ocké MC, Bueno-de-Mesquita HB, Pols MA, et al. (1997) The Dutch EPIC food frequency questionnaire. II. Relative validity and reproducibility for nutrients. Int J Epidemiol 26, Suppl. 1, S49-S58. 
21. NEVO Foundation (editor) (1996) Dutch Food Composition Database 1996 (NEVO) [In Dutch]. Den Haag: Voorlichtingsbureau voor de voeding.

22. Hertog MGL, Hollman PCH \& Katan MB (1992) Content of potentially anticarcinogenic flavonoids of 28 vegetables and 9 fruits commonly consumed in the Netherlands. J Agric Food Chem 40, 2379-2383.

23. Hertog MGL, Hollman PCH \& van de Putte B (1993) Content of potentially anticarcinogenic flavonoids of tea infusions, wines, and fruit juices. J Agric Food Chem 41, 1242-1246.

24. Arts IC, van De Putte B \& Hollman PC (2000) Catechin contents of foods commonly consumed in The Netherlands. 2 . Tea, wine, fruit juices, and chocolate milk. J Agric Food Chem 48, 1752-1757.

25. Arts IC, van de Putte B \& Hollman PC (2000) Catechin contents of foods commonly consumed in The Netherlands. 1. Fruits, vegetables, staple foods, and processed foods. J Agric Food Chem 48, 1746-1751.

26. Milder IE, Arts IC, van de Putte B, et al. (2005) Lignan contents of Dutch plant foods: a database including lariciresinol, pinoresinol, secoisolariciresinol and matairesinol. $\mathrm{Br} J$ Nutr 93, 393-402.

27. NEVO-online (2013) NEVO-online version 2013/4.O Bilthoven, the Netherlands: RIVM. http://nevo-online.rivm.nl

28. Pols MA, Peeters PH, Ocke MC, et al. (1997) Estimation of reproducibility and relative validity of the questions included in the EPIC Physical Activity Questionnaire. Int J Epidemiol 26, Suppl. 1, S181-S189

29. Wareham NJ, Jakes RW, Rennie KL, et al. (2003) Validity and repeatability of a simple index derived from the short physical activity questionnaire used in the European Prospective Investigation into Cancer and Nutrition (EPIC) study. Public Health Nutr 6, 407-413.

30. Van der Zee KI and Sanderman R (editors) (1993) Het meten van de algemene gezondheidstoestand met de RAND-36: een handleiding (Measuring the General Health Status with the RAND-36: A Manual). Groningen, the Netherlands: Noordelijk Centrum voor Gezondheidsvraagstukken.

31. Ware JEJ \& Sherbourne CD (1992) The MOS36-item shortform health survey (SF-36). I. Conceptual framework and item selection. Med Care 30, 473-483.

32. Willett W (editor) (1998) Nutritional Epidemiology, 2nd ed. New York, NY: Oxford University Press

33. Crichton GE, Bryan J \& Murphy KJ (2013) Dietary antioxidants, cognitive function and dementia - a systematic review. Plant Foods Hum Nutr 68, 279-292.

34. Rafnsson SB, Dillis V \& Trichopoulou A (2013) Antioxidant nutrients and age-related cognitive decline: a systematic review of population-based cohort studieds. Eur J Nutr 52, $1553-1567$

35. Li FJ, Shen L \& Ji HF (2012) Dietary intakes of vitamin E, vitamin $C$, and $\beta$-carotene and risk of Alzheimer's disease: a meta-analysis. J Alzheimers Dis 31, 253-258.
36. Marangon K, Herbeth B, Lecomte E, et al. (1998) Diet, antioxidant status, and smoking habits in French men. Am J Clin Nutr 67, 231-239.

37. Laurin D, Masaki KH, Foley DJ, et al. (2004) Midlife dietary intake of antioxidants and risk of late-life incident dementia: the Honolulu-Asia Aging study. Am J Epidemiol 159, 959-967.

38. Yaffe K, Clemons TE, McBee WL, et al. (2004) Impact of antioxidants, zinc, and copper on cognition in the elderly: a randomized, controlled trial. Neurology 63, 1705-1707.

39. Kang JH, Cook NR, Manson JE, et al. (2009) Vitamin E, vitamin $\mathrm{C}, \quad \beta$-carotene, and cognitive function among women with or at risk of cardiovascular disease: the Women's Antioxidant and Cardiovascular study. Circulation 119, 2772-2780.

40. Petersen RC, Thomas RG, Grundman M, et al. (2005) Vitamin $\mathrm{E}$ and donepezil for the treatment of mild cognitive impairment. $N$ Engl J Med 352, 2379-2388.

41. Harrison FE (2012) A critical review of vitamin C for the prevention of age-related cognitive decline and Alzheimer's disease. J Alzheimers Dis 29, 711-726.

42. Johnson EJ (2014) Role of lutein and zeaxanthin in visual and cognitive function throughout the lifespan. Nutr Rev 72, 605-612.

43. Johnson EJ (2012) A possible role for lutein and zeaxanthin in cognitive function in the elderly. Am J Clin Nutr 96, 1161S-1165S

44. Nooyens ACJ, van Gelder BM \& Verschuren WMM (2008) Smoking and cognitive decline among middle-aged men and women: the Doetinchem Cohort Study. Am J Public Health 98, 2244-2250.

45. Milder IE, Feskens EJ, Arts IC, et al. (2005) Intake of the plant lignans secoisolariciresinol, matairesinol, lariciresinol, and pinoresinol in Dutch men and women. $J$ Nutr 135, 1202-1207.

46. Heinonen S, Nurmi T, Liukkonen $\mathrm{K}$, et al. (2001) In vitro metabolism of plant lignans: new precursors of mammalian lignans enterolactone and enterodiol. J Agric Food Chem 49, 3178-3186.

47. Milder IEJ (2007) Lignan Intake in The Netherlands and Its Relation with Mortality. Wageningen: Wageningen University.

48. Joseph JA, Shukitt-Hale B, Casadesus G, et al. (2005) Oxidative stress and inflammation in brain aging: nutritional considerations. Neurochem Res 30, 927-935.

49. Cornwell T, Cohick W \& Raskin I (2004) Dietary phytoestrogens and health. Phytochemistry 65, 995-1016.

50. Kreijkamp-Kaspers S, Kok L, Grobbee DE, et al. (2007) Dietary phytoestrogen intake and cognitive function in older women. I Gerontol A Biol Sci Med Sci 62, 556-562.

51. Nooyens AC, Bueno-de-Mesquita HB, van Gelder BM, et al. (2014) Consumption of alcoholic beverages and cognitive decline at middle age: the Doetinchem Cohort Study. Br J Nutr 111, 715-723. 